\title{
KLF4 inhibits colorectal cancer cell proliferation dependent on NDRG2 signaling
}

\author{
YONGZHENG MA ${ }^{1 *}$, LIN WU $^{1 *}$, XUEWU LIU ${ }^{2}$, YUQIAO XU ${ }^{3}$, WEI SHI ${ }^{4}$, \\ YE LIANG $^{1,5}$, LIBO YAO ${ }^{1}$, JIANYONG ZHENG ${ }^{6}$ and JIAN ZHANG ${ }^{1}$
}

\author{
${ }^{1}$ The State Key Laboratory of Cancer Biology, Department of Biochemistry and Molecular Biology, \\ The Fourth Military Medical University; ${ }^{2}$ Department of Pathogenic Biology, The Fourth Military Medical University; \\ ${ }^{3}$ The State Key Laboratory of Cancer Biology, Department of Pathology, The Fourth Military Medical University; \\ ${ }^{4}$ The State Key Laboratory of Cancer Biology, Department of Urology, Xijing Hospital, The Fourth Military \\ Medical University; ${ }^{5}$ Cadet Brigade, The Fourth Military Medical University; ${ }^{6}$ The State Key Laboratory of \\ Cancer Biology, Department of Gastrointestinal Surgery, Xijing Hospital, The Fourth Military \\ Medical University, Xi'an, Shaanxi 710032, P.R. China
}

Received February 7, 2017; Accepted May 2, 2017

DOI: $10.3892 /$ or.2017.5736

\begin{abstract}
Krüppel-like factor 4 (KLF4) is a zinc finger transcription factor, which was confirmed as a tumor suppressor gene in colorectal cancers. KLF4 inhibits colorectal cancer cells proliferation through upregulating $\mathrm{p} 21^{\mathrm{WAF} 1 / \mathrm{Cip} 1}$ and downregulating cyclin D1. We firstly reported that N-Myc downstream regulated gene 2 (NDRG2) was a novel tumor suppressor gene in multiple cancers, such as glioma, breast cancer and colorectal cancer. Herein, we provide novel evidence that KLF4 can transcriptionally activate $N D R G 2$ by binding with $N D R G 2$ promoter. With MTT assay, EdU staining, colony formation assay and xenograft mouse model, we confirmed that KLF4 inhibited colorectal cancer cell proliferation and tumorigenesis dependent on NDRG2. Finally, with tissue array analysis, we found a positive correlation of combined detection of KLF4/NDRG2 co-expression with TNM grades and differentiation levels of colorectal cancer. Lower expression of KLF4 and NDRG2 in colorectal cancer patients was correlated with poor overall survival. Thus, KLF4 inhibited the prolifera-
\end{abstract}

Correspondence to: Professor Jian Zhang, The State Key Laboratory of Cancer Biology, Department of Biochemistry and Molecular Biology, The Fourth Military Medical University, Xi'an, Shaanxi 710032, P.R. China

E-mail: biozhangj@fmmu.edu.cn

Professor Jianyong Zheng, The State Key Laboratory of Cancer Biology, Department of Gastrointestinal Surgery, Xijing Hospital, The Fourth Military Medical University, Xi'an, Shaanxi 710032, P.R. China

E-mail: zhjy68@163.com

*Contributed equally

Key words: KLF4, NDRG2, colorectal cancer, proliferation, tumorigenesis tion of colorectal cancer cells dependent on NDRG2 signaling, which provides a novel strategy for therapy and early diagnosis of colorectal cancer.

\section{Introduction}

Colorectal cancer is one of the most common malignant tumors worldwide. In the past few years, the incidence and mortality of colorectal cancer increased rapidly and the onset age is much younger (1). It is promising that therapeutic options for patients have increased substantially, including earlier diagnosis and treatments such as surgery, radiotherapy, and chemotherapy (2). However, many colorectal cancers still remain incurable due to late stages. Therefore, prevention of progression and early metastasis become critical for colorectal cancer treatment.

Evidence indicates that several transcription factors can suppress colorectal cancer cell proliferation or migration successfully. High-mobility group AT-hook 2 (HMGA2) could induce the expression of Slug and promote EMT, migration, invasion, and proliferation of colorectal cancer cells (3). Inhibition of transcription factor Sp1 could suppress the growth of colorectal cancer stem cell and induce apoptosis (4). Noticeably, recent studies found that Krüppel-like factor 4 (KLF4) had important roles in suppressing colorectal cancer proliferation through upregulating $\mathrm{p} 21^{\mathrm{WAF} 1 / \mathrm{Cip} 1}$ and downregulating cyclin D1 (5). Overexpression of KLF4 in colorectal cancer cell line RKO could reduce the tumorigenesis ability. Evans showed that KLF4 was acetylated by p300/CBP to bind with $\beta$-catenin/TCF complex, and inhibited the proliferation effect induced by $\beta$-catenin (6). We are very curious whether there are other mechanisms of KLF4 in the suppression effect during colorectal cancer progression.

N-Myc downstream-regulated gene 2 (NDRG2) was first cloned in our laboratory (7). We confirmed that NDRG2 was a novel tumor suppressor, with decreased expression in colorectal tumors and other types of tumor tissues $(6,8)$. It has 
been indicated that NDRG2 was able to promote cell differentiation and suppress tumor cell proliferation. Our previous work found that NDRG2 can be transcriptionally regulated by p53, HIF-1 $\alpha$ and c-Myc (9-12). To better understand the function and regulation mechanism of NDRG2, in this study, we analyzed whether KLF4 could regulated NDRG2 expression in colorectal cancer model. There was three potential KLF4 binding sites in NDRG2 promoter predicted by MatInspector software analysis. It had been reported that KLF4 activated NDRG2 expression via binding with NDRG2 promoter. In our assay, we confirmed a novel binding site of KLF4 within $N D R G 2$ promoter that KLF4 could transcriptionally activate $N D R G 2$ using luciferase reporter analysis. With in vitro and in vivo analysis, we confirmed that KLF4 could suppress colorectal cancer cell proliferation depending on NDRG2 signaling. In colorectal cancer tissue array, expression level of KLF4 and NDRG2 was significantly correlated with the overall survival rate. Our data demonstrated that KLF4 inhibited colorectal cancer proliferation through transcriptional activation of $N D R G 2$.

\section{Materials and methods}

Cell culture. Two colorectal cancer cell lines, HT-29 and HCT-116 were grown and maintained in McCoy's 5a medium with $10 \%$ fetal bovine serum, respectively. HeLa and HEK-293T cells were also grown in Dulbecco's modified Eagle's medium (DMEM) with $10 \%$ fetal bovine serum. Cells were maintained at $37^{\circ} \mathrm{C}$ humidified incubator with $5 \% \mathrm{CO}_{2} / 95 \%$ air. All cell lines were sub-cultured at 3-day intervals. We purchased the HT-29 and HCT-116 cell lines from the American Type Culture Collection (ATCC). The cell lines were sub-cultured and stored by our research team and we have confirmed the genetic background through STR analysis.

Plasmid constructs. The human NDRG2 promoter was amplified from BAC clone RP11-998D10 (The Children's Hospital of Philadelphia, Philadelphia,PA, USA). The amplicon was cloned into the pGL3-basic vector to generate the pGL3-NDRG2-luc plasmid. Various truncations of the $N D R G 2$ promoter were generated with PCR by using pGL3-NDRG2-luc plasmid as template. The KLF4 was amplified from HT-29 cDNA. The resulting amplicon was cloned into the pcDNA3.1(+) and pFLAG-CMV vector to generate the pcDNA3.1-KLF4 and pFLAG-KLF4 vector. All the constructed plasmids were sequenced correctly.

Real-time PCR. Total RNA was isolated from parental cells or stable clones using TRIzol reagent (Takara, Dalian, China) according to the protocol. After reverse transcription, the resulting cDNA was used as the template for real-time PCR analysis. Real-time PCR was performed on an ABI 7500 system (Applied Biosystems). GAPDH was used as an internal control. Real-time PCR primers were designed using Primer Express v3.0 Software, and the sequences were: $N D R G 2$ forward primer: 5'-GAGATATGCTCTTAACCACCCG-3', NDRG2 reverse primer: 5'-GCTGCCCAATCCATCCAA-3'; GAPDH forward primer: 5'-TTCGACAGTCAGCCGCATCT TCTT-3', GAPDH reverse primer: 5'-CAGGCGCCAATAC GACCAAATC-3'. The PCR reaction consisted of $12.5 \mu \mathrm{l}$ of
SYBR Green PCR Master Mix, 300 nM each for forward and reverse primers, and $1.5 \mu \mathrm{g}$ template cDNA in a total volume of $25 \mu \mathrm{l}$. Thermal cycling conditions were: $95^{\circ} \mathrm{C}$ for $5 \mathrm{~min}$, followed by 40 cycles of $95^{\circ} \mathrm{C}$ for $30 \mathrm{sec}$ and $60^{\circ} \mathrm{C}$ for $30 \mathrm{sec}$.

Western blot analysis. Cells were collected from 6-well plates, and lysed in lysis buffer $(0.05 \mathrm{M}$ Tris- $\mathrm{HCl} \mathrm{pH} 7.4$, $0.15 \mathrm{M} \mathrm{NaCl}, 0.25 \%$ deoxycholic acid, $1 \%$ Nonidet P-40 (NP-40), $1 \mathrm{mM}$ EDTA, $1 \mathrm{mM}$ phenylmethylsulfonyl fluoride, $1 \mathrm{mg} / \mathrm{ml}$ aprotinin and $1 \mathrm{mg} / \mathrm{ml}$ leupeptin). Protein concentrations were measured using the Bicinchoninic acid (BCA) protein assay (Pierce, Rockford, IL, USA). Western blot analysis was carried out with standard protocol using nitrocellulose (NC) membranes (Amersham Biosciences). For the immunoblotting, the $\mathrm{NC}$ membranes were incubated with following primary antibodies: anti-NDRG2 (HPA002896; Sigma, St. Louis, MO, USA), anti-KLF4 (Cell Signaling Technology, \#4308), anti-p21 (Cell Signaling Technology, \#2947), anti-Cyclin D1 (Cell Signaling Technology, \#2926), anti-NDRG2 (Cell Signaling Technology, \#5667), and anti$\beta$-actin antibodies (Cell Signaling Technology, \#4970). Then, blots were incubated with horseradish peroxidase-conjugated secondary antibodies (Promega), and detected using the chemiluminescence method.

Luciferase reporter gene assays. HeLa cells were cultured in DMEM (with 10\% FBS) in 96-well plates with density of $1 \times 10^{4}$ cells/well overnight. NDRG2 reporter vectors including WT, and truncated mutants co-transfected with pcDNA3.1KLF4 using Lipofectamine-2000 (Invitrogen) for $48 \mathrm{~h}$. pRL-CMV plasmid was transfected to each well to monitor the transfection efficiency. The luciferase activities of reporter vectors were determined using the Dual-Luciferase reporter assay system (Promega).

Methyl thiazolyl tetrazolium (MTT) assay. All the parental cells and the stable clones were seeded separately with $1 \times 10^{4}$ cells/well in 96-well plates containing $200 \mu \mathrm{l} \mathrm{McCoy's}$ 5a medium (with 10\% FBS) and cultured for 5 days. Five wells from each group were selected for the MTT (Sigma) assay each day. After incubated with MTT for $4 \mathrm{~h}, 150 \mu \mathrm{l}$ of DMSO (Sigma) was added to each well. The percentage of viable cells was detected by measuring the absorbance at $490 \mathrm{~nm}$ on multiscanner reader (TECAN-spectra mini Grodig).

EdU assay. EdU staining was performed according to the instruction. Cells were grown in 24-well plate containing McCoy's 5a medium with 10\% FBS. After $6 \mathrm{~h}$ incubation with EdU (Rui Bo Co., Guangzhou, China), cells were fixed with $4 \%$ paraformaldehyde for $15 \mathrm{~min}$ at room temperature, and permeabilized with $0.5 \%$ Triton $\mathrm{X}-100$ for $10 \mathrm{~min}$, then stained with 1X Apollo ${ }^{\circledR}$ for $30 \mathrm{~min}$ at room temperature. Finally, DAPI was used for nuclear staining. Positively stained cells were counted in five randomly selected visual fields.

Plate colony formation assay. For colony formation assays, 500 cells were seeded into 60-mm dishes with McCoy's 5a medium (with $10 \%$ FBS). After 2 weeks, the resulting colonies containing at least 50 cells were fixed with methanol and stained with Giemsa (Sigma). Only clear colonies were 

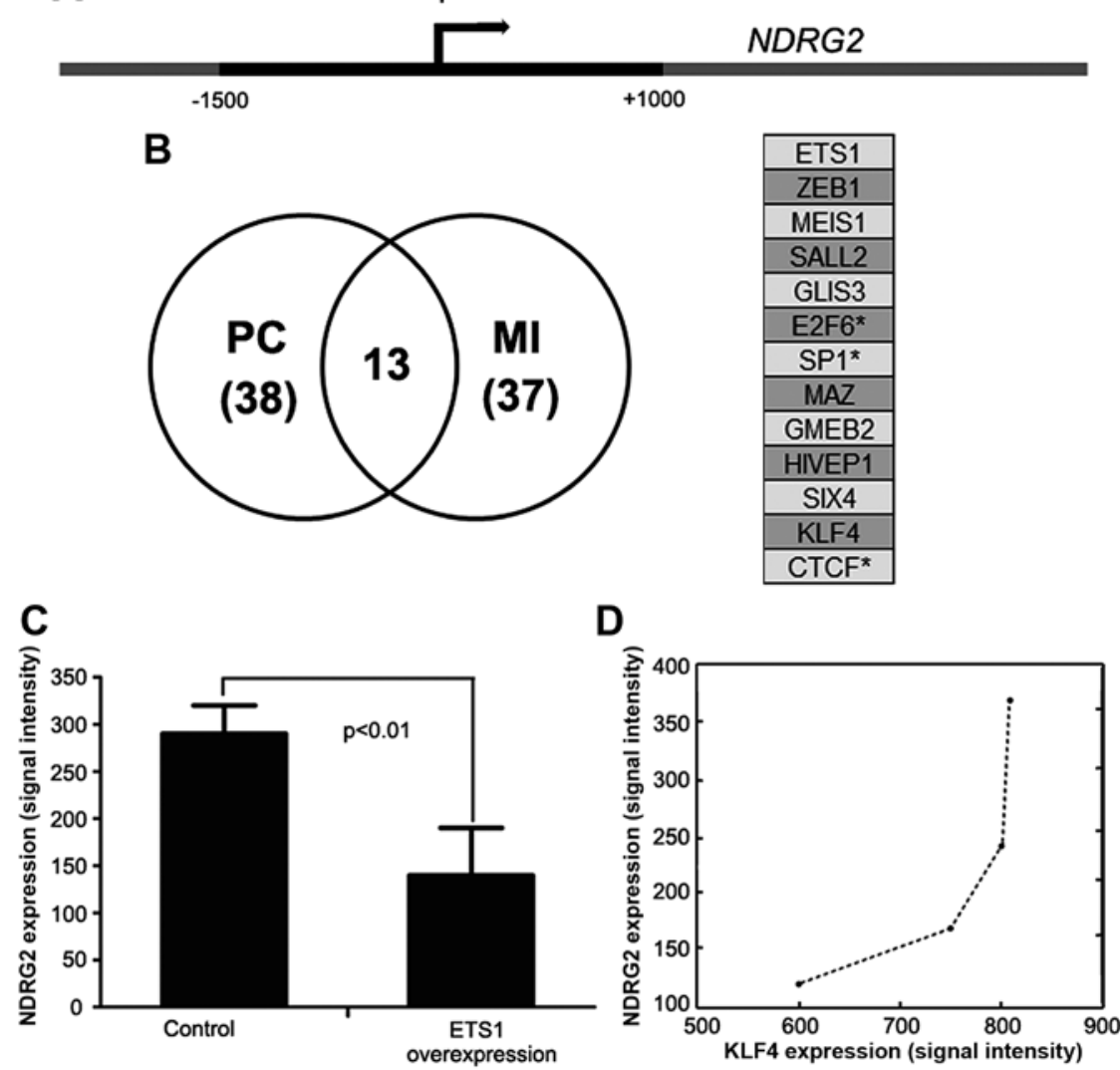

Figure 1. Prediction of TFs regulating NDRG2 expression. (A) The active region around the transcription start site of NDRG2. (B) Predicted TFs regulating $N D R G 2$ expression. Left panel, Venn diagram of TFs correlated with NDRG2 expression. PC, Pearson correlation. MI, mutual information. Right panel, predicted 13 TFs. TFs reported or annotated to regulate NDRG2 expression. (C) ETS1 suppress NDRG2 expression in HeLa cells. Expression data was obtained from published microarray dataset GSE21129. (D) NDRG2 expression positively correlated with KLF4 expression. Expression values were obtained from GSE4410 (Spearman correlation, $r=1, \mathrm{p}=0.083$ ).

counted. Assays were conducted in duplicate in three independent experiments.

Tumorigenicity in nude mice. The male nude mice weighing 15-20 g and 4-6 weeks of age were purchased from laboratory animal research center of the Fourth Military Medical University. Mice were separated into four groups of five mice per group. The cells $\left(5 \times 10^{6}\right)$ were inoculated subcutaneously into the right flank of the nude mice to establish xenografts. Tumor sizes were measured every 4 days with a slide caliper and calculated using the formula: length $\mathrm{x}$ width ${ }^{2} / 2$. Animals were sacrificed 20 days after inoculation. All animal studies were performed in accordance with the international guidelines for the care and treatment of laboratory animals.

Immunohistochemistry. The study of human samples was approved by Institutional Ethics Committee (IEC) of the First Affiliated Hospital of Fourth Military Medical University, and an informed consent was signed by the patients prior to the study project. All procedures for study of human samples were performed according to the relevant guidelines and regulations of the First Affiliated Hospital of Fourth Military Medical University. Human colon cancer tissues were collected between year 2008 and 2013 in First Affiliated Hospital of Fourth Military Medical University. Tumor tissues were fixed with formalin and embedded in paraffin. The samples were incubated with polyclonal antibodies of NDRG2 and monoclonal antibody of KLF4, respectively. Then the sections were incubated with secondary antibody for $1 \mathrm{~h}$ at room temperature. After washing, the sections were incubated with DAB (ZSGBBiotechnology, Beijing, China), and lightly counterstained with hematoxylin, then observed under a photomicroscope.

Evaluation of IHC staining. Staining was evaluated by scanning the entire tissue specimen under appropriate magnification. Score of IHC staining was described previously. The criteria for a sample to be scored was set to the presence of at least one core containing 50 intact tumor cells. The internal background was discarded. Based on previous study, the expression of NDRG2 was mainly localized in the cytoplasm, so we calculated the cytoplasm expression of NDRG2 as positive. The median was used as cutoff to define the positive cases, and samples with below $5 \%$ positively stained cells were considered negative. The staining grade was stratified as absent (0 score), weak (1-4 score), moderate (5-8 score) or strong (9-12 score).

Statistical analysis. Data were generally expressed as mean \pm standard error values. Groups of data were compared by analysis of variance (ANOVA) and post hoc analysis using Student-Keuls method. The statistics were performed with SPSS 16.0 software. A value of $\mathrm{P}<0.05$ was considered to indicate a statistically significant difference. 


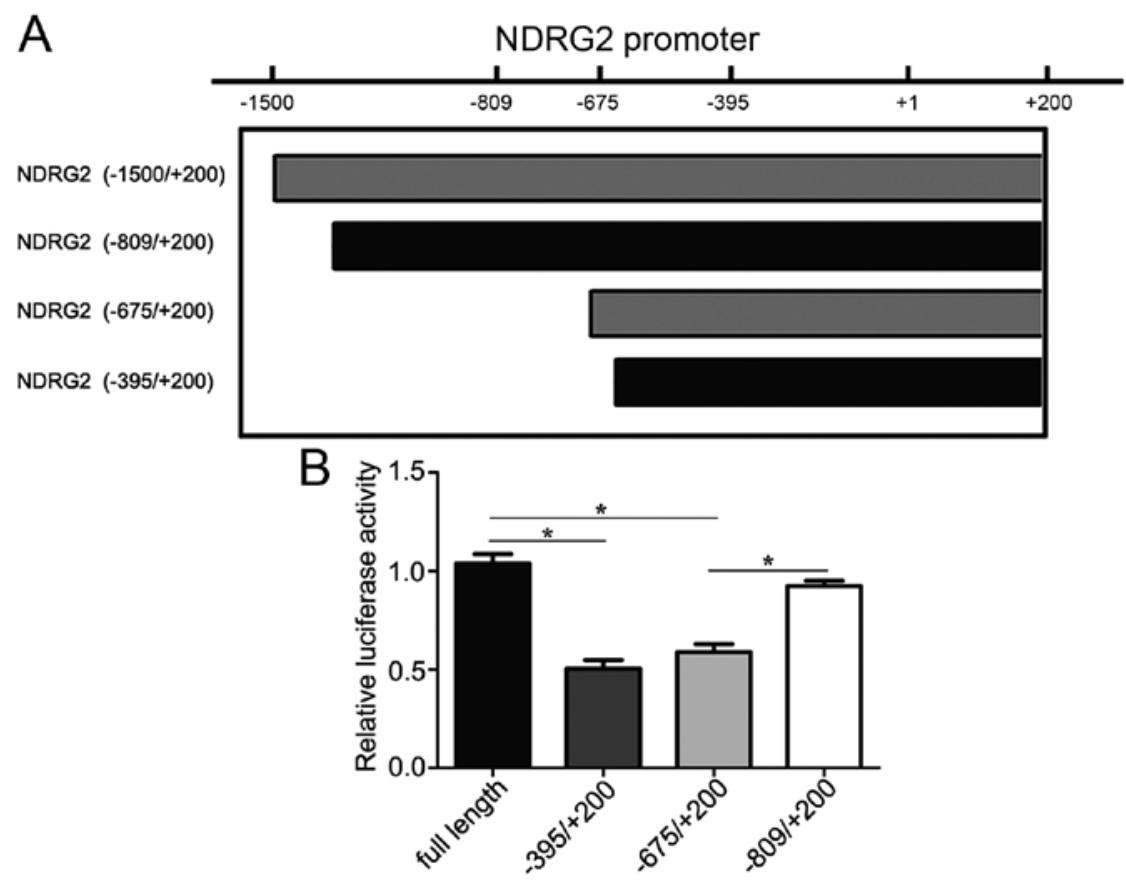

Figure 2. KLF4 transcriptionally activates NDRG2 expression. (A) Structures of $N D R G 2$ promoter and various truncations of $N D R G 2$ promoter, numbers on the left side of the bars indicate the relative position of the mutants. (B) $N D R G 2$ promoter activities of the different truncations. HeLa cells were transfected with these different fragments of the NDRG2 promoter, KLF4 and luciferase, respectively. The relative luciferase activity (firefly luciferase/Renilla luciferase) was analyzed $48 \mathrm{~h}$ later. The relative luciferase activity (firefly luciferase/Renilla luciferase) was analyzed $48 \mathrm{~h}$ later. All the assays were duplicated in at least three independent experiments. The results are shown as the mean \pm SD. ${ }^{*} \mathrm{P}<0.01$ and ${ }^{* *} \mathrm{P}<0.05$ compared with control.

\section{Results}

Prediction of transcription factors regulating NDRG2 expression. To further explore the function and transcription regulation mechanism of $N D R G 2$, we adopted two independent resources to predict transcription factors regulating NDRG2 expression, including transcription factor (TF) binding site and gene expression correlation. Combination of these two independent resources has been shown as an effective way to predict the TFs regulating the transcription of a particular gene (13). First, the active transcription region bound by acetylated $\mathrm{H} 3 \mathrm{~K} 27$ at the transcription start site of $N D R G 2$ was extracted from UCSC genome browser (Fig. 1A). Then, the active 2500 -bp region $(-1500 \mathrm{bp}$ to $+1000 \mathrm{bp})$ was scanned for transcription factor binding sites by MatInspector with defaulting parameters. There were 298 transcriptional factors predicted to regulate $N D R G 2$ expression. Generally, not all those predicted transcriptional factors were involved in $N D R G 2$ expression regulation. Some factors were false positively predicted. To improve prediction precision, gene expression correlation was integrated. If a transcription factor is involved in regulation of $N D R G 2$ expression, its expression variation might lead to $N D R G 2$ expression change. Therefore a significant expression correlation can be observed between the TFs and NDRG2. The microarray datasets GSE2350 were employed to find the transcription factors whose expression levels were significantly correlated with $N D R G 2$ expression. After removing the TFs not shown in those microarray datasets, the remaining $139 \mathrm{TFs}$ were accessed for their expression correlation with $N D R G 2$ expression. We accessed linear correlation using Pearson correlation and non-linear correlation using mutual information.
As shown in Fig. 1B, the numbers of TFs having significant linear and non-linear correlation with $N D R G 2$ expression are 38 and 37, respectively. Only 13 TFs show significant correlation with NDRG2 expression regardless of expression correlation accessed with Pearson correlation or mutual information. As few of the TFs were reported to regulate $N D R G 2$ expression, it is hard to access the performance of our prediction. However, by combination of TF binding sites and expression correlation, we indeed predicted several TFs truly regulating $N D R G 2$ expression. For example, SP1 has been shown to be activated by TGF- $\beta$ signaling pathway (6), subsequently promoting NDRG2 expression (Fig. 1B, right panel). CTCF and E2F6 annotated to bind with the active region were also identified by our method (14). However, the transcriptional factor WT1 was excluded from candidates for the reason that its expression was not significantly correlated with $N D R G 2$ expression (15). Moreover, c-Myc was also excluded because its binding site was not identified at the active region by MatInspector. In these candidates, ETS1 expression has the most significant correlation with $N D R G 2$ expression. Whether ETS1 can regulate $N D R G 2$ expression was unclear.

NDRG2 plays as a tumor suppressor gene in various types of malignant cancers. It has been reported that NDRG2 also inhibited the proliferation and metastasis of ovarian cancer cells (9). Herein, we used the microarray dataset GSE21129 from ovarian cancer, and found that ectopic expression of ETS1 in HeLa cells was able to reduce NDRG2 expression, implying that ETS1 can directly or indirectly regulate NDRG2 expression (Fig. 1C). These results from different datasets suggested that our prediction was more reliable.

Among these 13 candidates, KLF4 was a crucial TF for intestinal epithelium differentiation (16), while $N D R G 2$ 

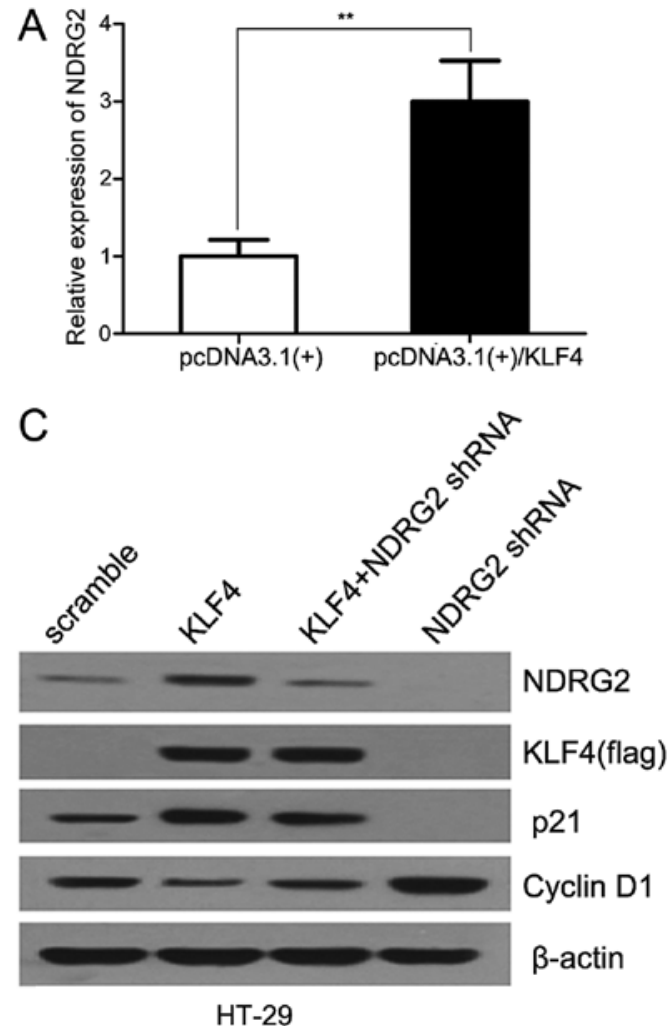
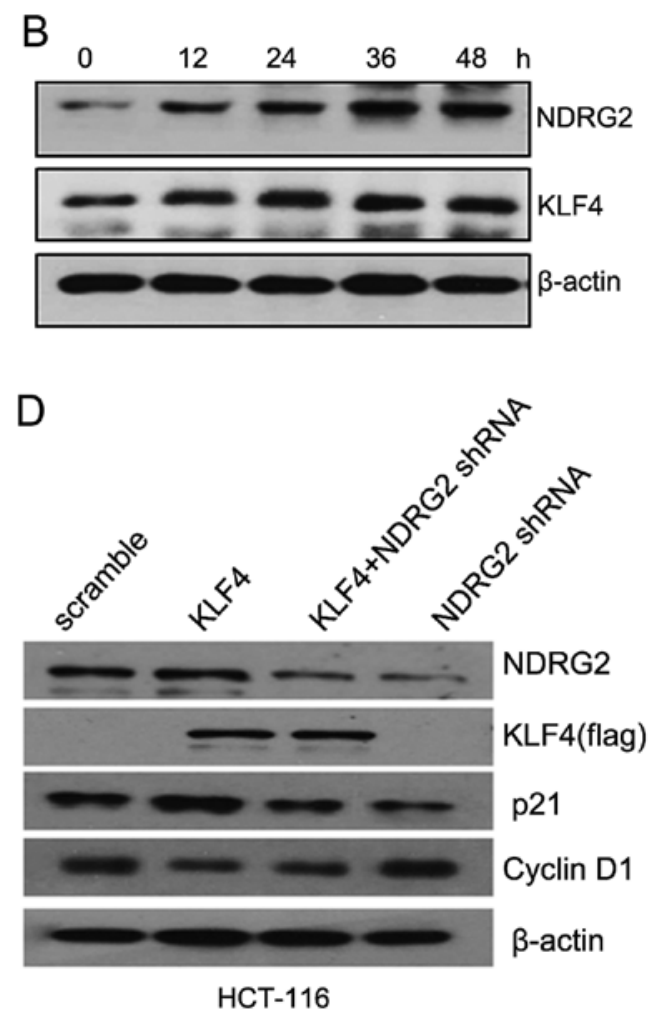

Figure 3. KLF4 induces NDRG2 expression in colorectal cancer cell lines. (A) Transcriptional activation of $N D R G 2$ expression assayed by Real-time PCR after ectopic expression of KLF4. (B) Level of KLF4 and NDRG2 expression by western blotting in time-dependent manner in HT-29 cells. (C) Western blotting for KLF4, NDRG2, p21, cyclin D1 in HT-29 cells. (D) Western blotting for KLF4, NDRG2, p21, cyclin D1 in HCT-116 cells. (C and D) Analysis was carried out after transfection of pcDNA3.1-KLF4 for $48 \mathrm{~h}$.

was upregulated during the process of intestinal epithelium differentiation (12), suggesting KLF4 might participate in upregulating $N D R G 2$ expression. Moreover, by analysis of the published microarray data GSE4410 (17), we observed a positively correlation between NDRG2 and KLF4 expression levels when colorectal epithelial cells were induced to differentiate by sodium butyrate (Spearman's correlation, $r=1$, $\mathrm{P}=0.083$ ) (Fig. 1D). Therefore, we chose KLF4 for further experiment validation.

KLF4 transcriptionally activates NDRG2 expression. To further explore the molecular mechanism of KLF4 regulating $N D R G 2$ expression, we constructed a series of different length of $N D R G 2$ promoter $(-1500 /+200 \mathrm{bp})$, including the truncations and mutants. HeLa cells were transfected with the NDRG2 promoter luciferase reporter gene vector and the plasmid of pcDNA3.1-KLF4. We detected the higher levels of NDRG2 promoter activity in the cells transfected with KLF4, but not in the control (luciferase vector only, data not shown) (Fig. 2A). Different transcriptional activities were detected in the truncations and mutants. Obviously, full length of NDRG2 promoter (-1500/+200 bp) exhibited the highest activity, and NDRG2 (-809/+200 bp) promoter showed almost the same transcriptional activity compared with the full length. Otherwise, NDRG2 (-675/+200 bp) exhibited suppressive promoter activity. Moreover, NDRG2 (-395/+200) showed the inhibitory transcriptional activity and was almost the same compared with NDRG2 (-675/+200 bp) (Fig. 2B). This result revealed that KLF4 transcriptionally regulated $N D R G 2$ expression through the promoter located between the region -809/-675 bp. It was reported that KLF4 transcriptionally regulated NDRG2 expression via binding to the promoter located between $-133 /+55$ (18). Our current study demonstrated a novel binding site for KLF4 within NDRG2 promoter. Simultaneously, we also determined that KLF4 induced the expression of NDRG2 both at mRNA and protein levels in a time-dependent manner in HT-29 cells (Fig. 3A and B). Our findings demonstrated the novel evidence that KLF4 transcriptionally activated NDRG2 via binding to its promoter.

KLF4 inhibits colorectal cancer cell proliferation through upregulation of NDRG2. To further elucidate the function of KLF4-NDRG2 signaling, we subsequently analyzed whether KLF4 inhibited the proliferation of colorectal cancer cells through upregulation of NDRG2. It has been reported that KLF4 could inhibit cancer cell proliferation via upregulating p21 expression and suppressing cyclin D1. In our study, as predicted, we found that KLF4 induced p21 expression and suppressed cyclin D1 in HT-29 cells. While shRNA-mediated downregulation of NDRG2 decreased p21 expression and enhanced cyclin D1, and attenuation of NDRG2 suppressed the modulation of $\mathrm{p} 21$ and cyclin D1 induced by KLF4 (Fig. 3C and D). To further confirm the function of KLF4NDRG2 signaling pathway, we also found that overexpression of KLF4 could inhibit the proliferation of HT-29 and HCT-116 cells, while shRNA-mediated attenuation of NDRG2 could rescue cancer cell proliferation inhibited by KL4. Our data showed that downregulation of NDRG2 could reverse the 

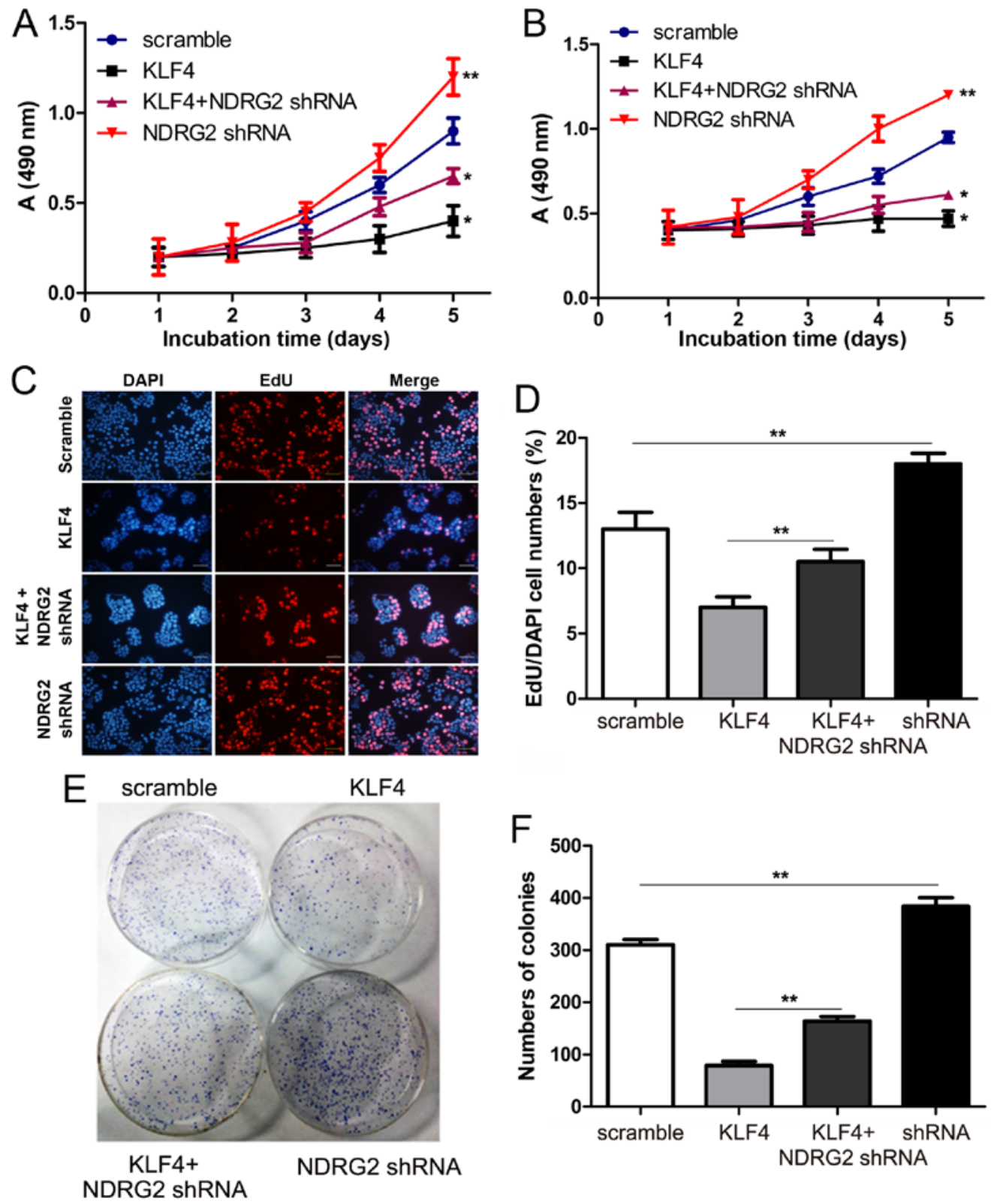

Figure 4. KLF4 inhibits the proliferation of colorectal cancer cell lines dependent on NDRG2 upregulation. (A) The growth of the HT-29 cells determined by MTT assay. (B) The growth of the HCT-116 cells determined by MTT assay (C) EdU staining in HT-29 cells. Proliferating cells were stained with EdU (red), and nuclei were counterstained with DAPI (blue) (scale bars $=40 \mathrm{~mm}$ ). (D) Colony formation assays in HT-29 cells. HT-29 cells were plated in 6-cm plates with media and incubated for 15 days before counting the number of foci. (D and F) Data represent means \pm SEM from three independent experiments conducted in triplicate. ${ }^{*} \mathrm{P}<0.05$ and ${ }^{* *} \mathrm{P}<0.01$ compared with control cells.

inhibitory effect of KLF4 in the two cell lines. Cell proliferation difference was much significant between KLF4 and KLF4 + NDRG2 shRNA groups for the time periods of 4 and 5 days (Fig. 4A and B), suggesting that KLF4 inhibited the proliferation of colorectal cancer cells dependent on NDRG2. Furthermore, EDU staining and colony formation assay also confirmed that KLF4 inhibited proliferation of colorectal cancer cell lines via upregulation of NDRG2 (Fig. 4C-F).

NDRG2 reverses the role of KLF4 inhibiting tumorigenesis in vivo. Next, we further evaluated the effect of KLF4-NDRG2 signaling inhibiting tumorigenesis in vivo. Colorectal cancer cells HT-29 with different expression levels of NDRG2 and KLF4, including HT-29-Scramble, HT-29-KLF4, HT-29-NDRG2
shRNA and HT-29-KLF4/NDRG2 shRNA, HT-29-Control, HT-29-NDRG2 and HT-29-NDRG2/KLF4 cells, were injected into nude mice respectively. Tumor size was evaluated every 4 days, and on day 20 , tumor mass was weighed.

In our study, the mice injected with HT-29/KLF4 showed a statistically significant decrease in tumor size and tumor mass compared with the control group. The mice injected with HT-29-KLF4/ NDRG2 shRNA showed a slight decrease in tumor size and tumor mass (Fig. 5A and B). Moreover, the mice injected with HT-29-NDRG2 and HT-29-KLF4/NDRG2 decreased the tumor size and tumor mass significantly (Fig. 5C and D). These data demonstrated that KLF4 inhibited the tumorigenesis of colorectal cancer via upregulation of NDRG2 expression in vivo. 
Table I. Statistical results of the immunohistology.

\begin{tabular}{|c|c|c|c|c|c|c|c|}
\hline \multirow[b]{2}{*}{ Total } & \multirow[b]{2}{*}{$\mathrm{n}$} & \multicolumn{2}{|c|}{ KLF4 } & \multirow[b]{2}{*}{ P-value } & \multicolumn{2}{|c|}{ NDRG2 } & \multirow[b]{2}{*}{ P-value } \\
\hline & & - & \pm to ++ & & - & \pm to ++ & \\
\hline \multicolumn{8}{|l|}{ Sex } \\
\hline Male & 62 & 33 & 29 & $0.231^{\mathrm{a}}$ & 27 & 35 & $0.520^{\mathrm{a}}$ \\
\hline Female & 39 & 25 & 14 & & 22 & 17 & \\
\hline \multicolumn{8}{|l|}{ Age } \\
\hline$<60$ & 53 & 22 & 31 & $0.428^{\mathrm{a}}$ & 20 & 33 & $0.315^{\mathrm{a}}$ \\
\hline$\geq 60$ & 48 & 36 & 12 & & 29 & 19 & \\
\hline \multicolumn{8}{|l|}{ WHO grade } \\
\hline I & 38 & 19 & 19 & $0.012^{\mathrm{b}}$ & 18 & 20 & $0.021^{\mathrm{b}}$ \\
\hline II & 51 & 29 & 22 & & 23 & 28 & \\
\hline III & 12 & 10 & 2 & & 8 & 4 & \\
\hline \multicolumn{8}{|c|}{ Differentiation status } \\
\hline Well & 59 & 33 & 26 & $0.035^{\mathrm{b}}$ & 29 & 30 & $0.014^{\mathrm{b}}$ \\
\hline Moderately & 36 & 20 & 16 & & 16 & 20 & \\
\hline Poor & 6 & 5 & 1 & & 4 & 2 & \\
\hline
\end{tabular}

${ }^{\text {aP}} \mathrm{P}-\mathrm{value}$ when expression levels were compared using Fisher's exact test. ${ }^{\mathrm{b}} \mathrm{P}$-value when expression levels were compared using Kruskal Wallis.

Decreased expression of KLF4 and NDRG2 correlates with poor overall survival of colorectal cancer patients. To investigate the clinical significance of KLF4 and NDRG2 expression in colorectal cancer patients, we used colorectal cancer tissue array with 101 colorectal cancer samples to analyze the survival correlation. The characteristics of the 101 colorectal cancer patients involved in the study cohort are shown in Table I. In the 101 colorectal cancer patients, there were 62 male $(61.4 \%)$ and 39 (38.6\%) female patients. The mean age was 64 years, with a range of 16-85. Tumor with well/moderately/poorly differentiated was $41(58.4 \%), 33(35.6 \%)$ and $6(6 \%)$, respectively. According to the International TNM (Tumor Node Metastasis) Classification, 38 (37.6\%), 51 (50.5\%), and 12 (11.9\%) of the 101 colorectal cancer patients were classified as TNM stages I, II, and III, respectively. In all samples, KLF4 and NDRG2 expression was correlated with TNM grades and differentiation levels of colorectal cancer (Table I).

With immunohistochemistry assay, expression of KLF4 was positively correlated with NDRG2 (Fig. 6A and B). We also examined the correlation of co-expression of KLF4 and NDRG2 with the overall survival rate. Kaplan-Meier survival curves were applied, and we found that reduced expression of KLF4 and NDRG2 had a significantly shorter survival time compared with those with a higher KLF4 and NDRG2 expression ( $\mathrm{p}<0.05$; Fig. 6C). Taken together, NDRG2 expression was positively correlated with KLF4, and higher NDRG2 expression was associated with better overall survival rate in colorectal cancer patients.

\section{Discussion}

The Krüppel-like factor (KLF) family members are transcription factors functioned in several biological processes. The members of KLF family have highly conserved zinc-finger domain, which can bind to similar DNA binding domain such as CACCC and GC-rich region (19-22). The SP/KLF transcription factor family member KLF4 is located in chromatin 9q31 (23), and is highly expressed in the epithelia of the skin, lungs and intestinal tract and other organs (24). KLF4 plays important roles in regulating multiple cellular processes including cell proliferation, differentiation, apoptosis, inflammation and also tumor formation. However, it is puzzling that KLF4 can play both oncogenic and tumor suppressive functions in different tissue types depending on regulation of various target genes. Over $70 \%$ breast cancers showed high expression level of KLF4, and upregulated KLF4 can enhance tumorigenesis, cell migration and cell invasion (25). On the contrary, decreased expression of KLF4 was found in colorectal cancer, gastric cancer, intestinal adenomas, and pancreatic ductal carcinoma, which suggested that KLF4 could function as a tumor suppressor gene, and correlate with inhibitory abilities of cell proliferation, invasion and tumorigenesis (26-29). KLF4 was identified as an independent predictor of survival and recurrence of colorectal cancer.

Previously our laboratory identified NDRG2 from normal human brain cDNA library with subtractive hybridization (7). It belonged to $N D R G$ gene family together with $N D R G 1$, $N D R G 3$, and $N D R G 4$, and was involved in cell stress, differentiation and proliferation (30). We and other laboratories confirmed that NDRG2 was a novel tumor suppressor gene with decreased expression in several tumor tissues and cancer cells such as breast cancer, glioma, and colorectal cancers (31-34). In a previous study, we found that lower expression of NDRG2 had strong proliferation and invasion abilities of colorectal cancer cells, also NDRG2 was a potential independent prognosis biomarker of human colorectal cancer (35). 

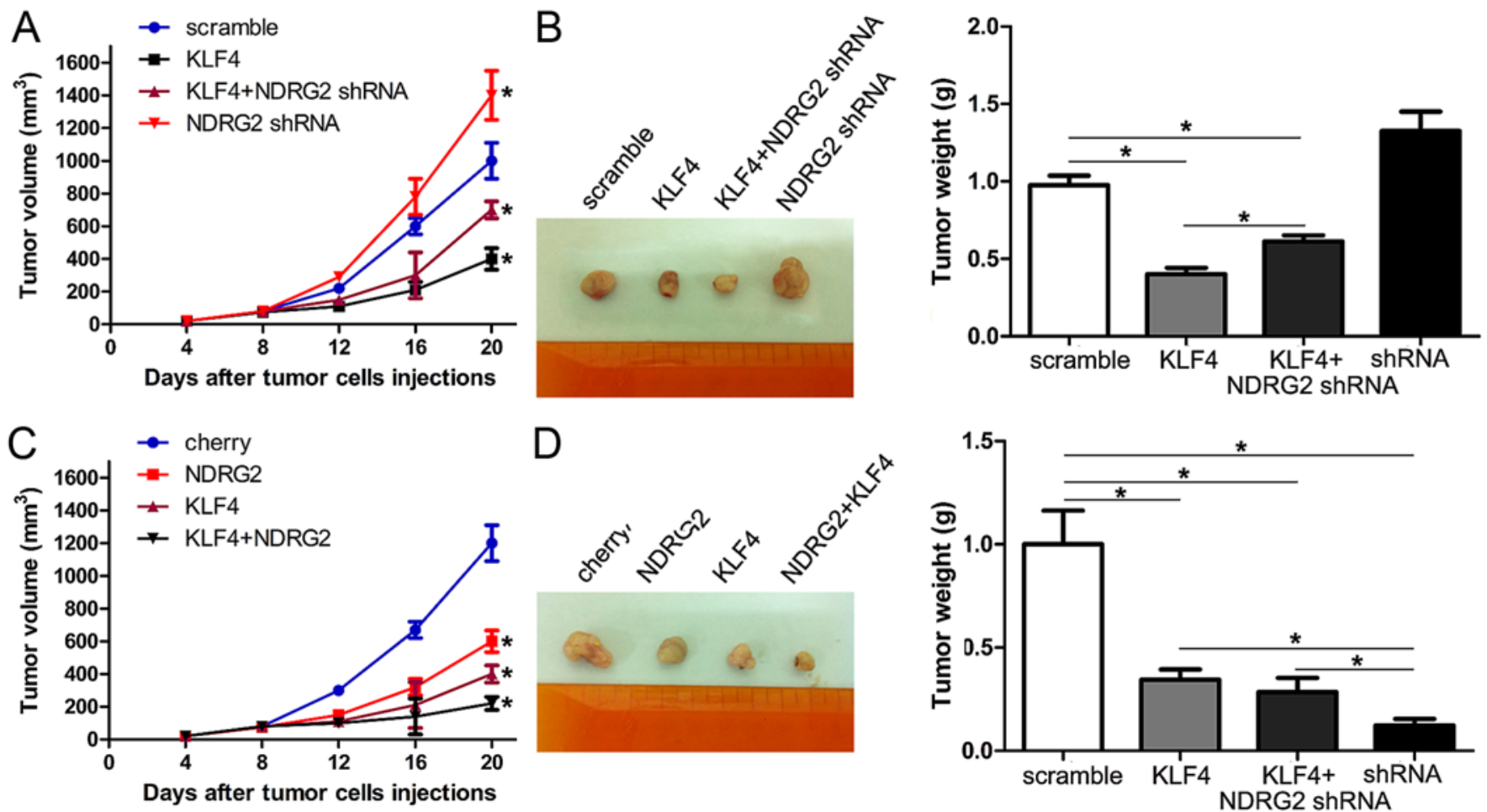

Figure 5. KLF4 suppresses tumorigenesis dependent on NDRG2 in vivo. (A) Tumor growth curve of the nude mice injected with HT-29-Scramble, HT-29-KLF4, HT-29-NDRG2 shRNA/KLF4 and HT-29-NDRG2 shRNA cells. Scale bar, $1 \mathrm{~cm}$. (B) Tumor size obtained from the nude mice with different types of HT-29 cells. (C) Tumor growth curve of the nude mice injected with HT-29-Control, HT-29-NDRG2, HT-29-KLF4 and HT-29-KLF4/NDRG2 cells. (D) Tumor size dissected from nude mice injected with different types of HT-29 cells. Scale bar, $1 \mathrm{~cm}$. The data are generated from three mice in three different groups, and analyzed at least three times. The data represent means \pm SEM. ${ }^{*} \mathrm{P}<0.05$ and ${ }^{* *} \mathrm{P}<0.01$ compared with control cells.

A

KLF4
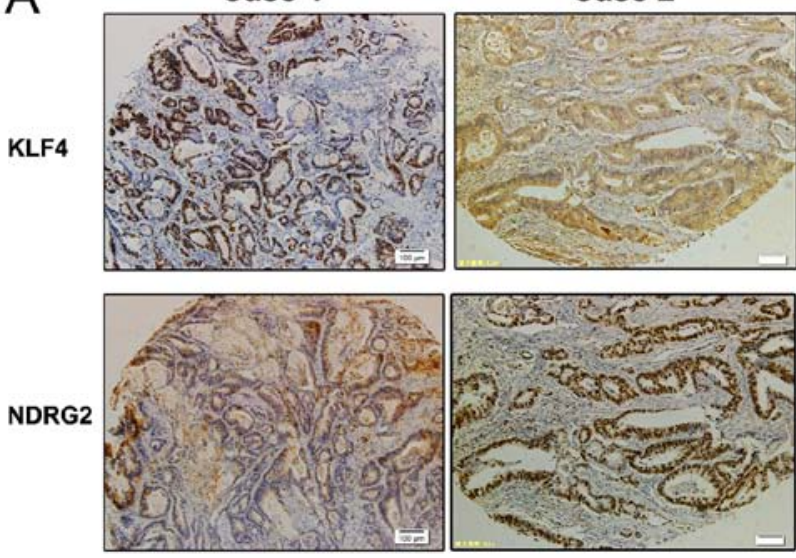

Case 2

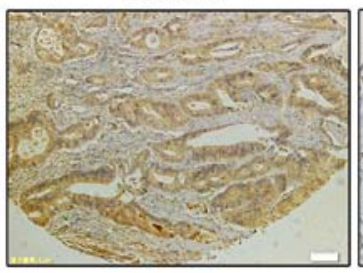

Case 3
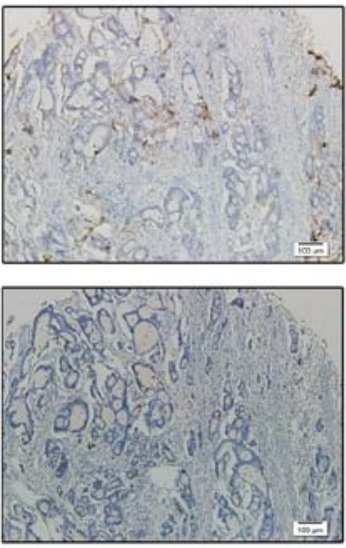

Case 4
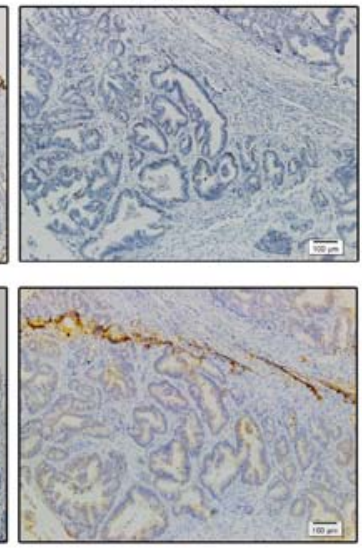

B

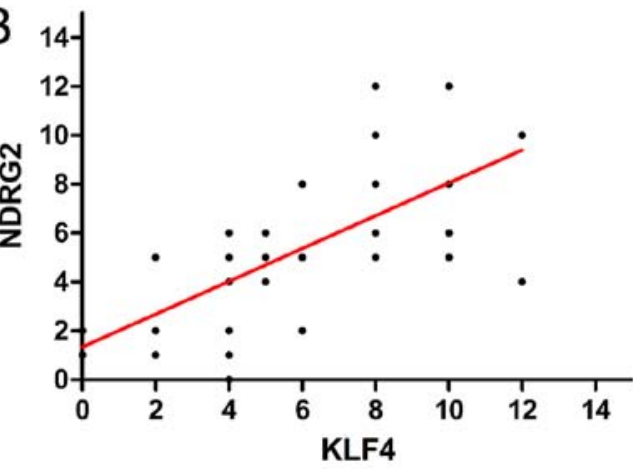

C

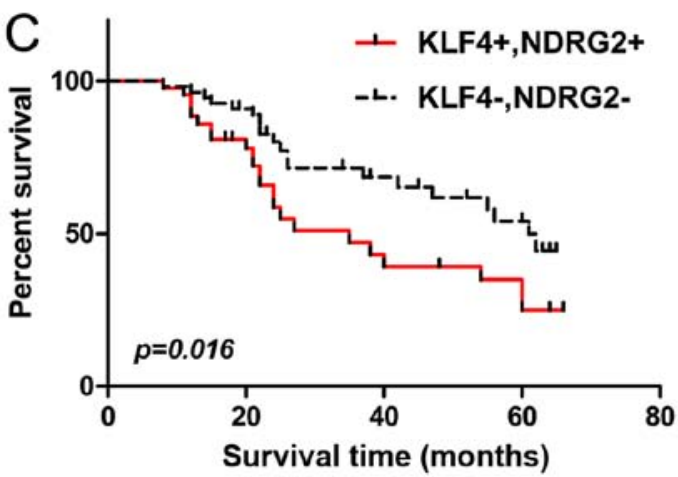

Figure 6. Correlation of KLF4 and NDRG2 expression with overall survival. (A) Immunohistochemical analysis of KLF4 and NDRG2 in the colorectal tumor array. Scale bar, $100 \mu \mathrm{m}$. (B) Correlation of KLF4 and NDRG2 expression with linear regression and Pearson's correlation significance in colorectal tumor analysis (ANOVA test, $\mathrm{P}=0.004$ ). (C) Kaplan-Meier survival curves of colorectal cancer patients with $\mathrm{KLF}^{+} / \mathrm{NDRG}^{+}\left(\mathrm{n}=50\right.$ ) expression and $\mathrm{KLF} 4 / \mathrm{NDRG}^{-}$ expression ( $\mathrm{n}=30)$. $\mathrm{P}=0.016$. 
In this study, we used bio-information analysis and found there were three potential KLF4 binding sites locating on $N D R G 2$ promoter (Fig. 1). Then we used reporter gene assay to explore whether KLF4 transcriptionally activated $N D R G 2$. We constructed different truncations based on NDRG2 promoter, and found KLF4 could upregulate NDRG2 promoter activity especially on $-809 /-675$ and $-395 /+200$ bp sites (Fig. 2), while $-133 /+55$ bp site had been reported previously, we confirmed a novel binding site of KLF4 on NDRG2 promoter. In NDRG2 promoter, there might be two different transcription start sites which were predicted with bio-information system, and this is a possible reason why our binding site of KLF4 on NDRG2 promoter is not the same.

To further explore the role of NDRG2 in KLF4 suppressing proliferation in colorectal cancer cells, we downregulated NDRG2 expression in HT29 cells with KLF4 overexpression, and performed in vitro biology experiments including MTT, EdU staining and colony formation assay. Results demonstrated that NDRG2 could abrogate the function of KLF4 by inhibiting colorectal cancer cell proliferation through the regulation of p21 and cyclin D1 in vitro (Fig. 4). As our previous report, NDRG2 overexpression could induce cell cycle arrest, which might be due to its regulation of $\mathrm{p} 21$ and cyclin D1 expression. Herein, we found that NDRG2 knockdown caused downregulation of p21 and upregulation of cyclin D1, which was consistent with our previous finding in cell cycle analysis (data not shown). Furthermore, in a nude mouse xenograft model, the tumor sizes and weight of KLF4 and shNDRG2 group were smaller compared with the control group (Fig. 5). All these results revealed that NDRG2 played an important role in KLF4 signaling of colorectal cancer proliferation inhibition.

Based on previous studies, KLF4 acts as a tumor suppressor gene and inhibites the proliferation of various types of tumor cells via different signaling pathway $(27,29)$. Moreover, NDRG2 inhibited cell proliferation through upregulation of p21, p27, and p53 $(6,8,9)$. As the signaling pathway of NDRG2 and KLF4 was only partially crossed, it is reasonable to understand the co-overexpression of KLF4 and NDRG2 could inhibit the proliferation of colorectal cancer cells more obviously than KLF4 or NDRG2, respectively.

Previous studies have demonstrated that KLF4 and NDRG2 are both predictors of survival and recurrence for colorectal cancer. It is not clear whether the association of KLF4/ NDRG2 combined expression could benefit us in prediction of better prognosis for patients with colorectal cancer. In the present study, we used a colorectal cancer tissue array with 101 colorectal cancer samples to analyze their expression with tumor prognosis. There was no significant association between KLF4/NDRG2 expression and sex or age at diagnosis (Table I). We observed that lower expression of KLF4 and NDRG2 was evident in human colorectal tissues compared with normal tissues, and it was greatly positively related with the TNM grades and differentiation level of colorectal cancer. KaplanMeier analysis revealed significant difference in prognosis depending on the status of KLF4/NDRG2 co-expression. $\mathrm{KLF}^{+} / \mathrm{NDRG}^{+}$had better overall survival than $\mathrm{KLF}^{-} \%$ NDRG2: However, further investigation in many more cases is still needed to evaluate the potential application value of KLF4/NDRG2 co-expression in clinical setting.
In conclusion, our data showed that KLF4 could transcriptionally upregulate $N D R G 2$ expression by binding with its promoter. NDRG2 downregulation could interrupt the function of KLF4 in suppressing colorectal cancer cell proliferation and tumorigenesis both in vitro and in vivo. In colorectal cancer tissue array, we found that a combined detection of KLF4/NDRG2 was positively related with TNM grades and differentiation levels. The co-expression of KLF4/NDRG2 may be beneficial in predicting the prognosis of colorectal cancer patients.

\section{Acknowledgements}

This work was supported by the National High-tech R\&D Program of China for Young Scientist (2014AA020517), and National Natural Science Foundation of China (nos. 81172292, 31571437, 81230043, 81372390 and 81421003).

\section{References}

1. Feagins LA, Souza RF and Spechler SJ: Carcinogenesis in IBD: Potential targets for the prevention of colorectal cancer. Nat Rev Gastroenterol Hepatol 6: 297-305, 2009.

2. Lamprecht SA and Lipkin M: Chemoprevention of colon cancer by calcium, vitamin D and folate: Molecular mechanisms. Nat Rev Cancer 3: 601-614, 2003.

3. Li Y, Zhao Z, Xu C, Zhou Z, Zhu Z and You T: HMGA2 induces transcription factor Slug expression to promote epithelialto-mesenchymal transition and contributes to colon cancer progression. Cancer Lett 355: 130-140, 2014.

4. Zhao Y, Zhang W, Guo Z, Ma F, Wu Y, Bai Y, Gong W, Chen Y, Cheng T, Zhi F, et al: Inhibition of the transcription factor Sp1 suppresses colon cancer stem cell growth and induces apoptosis in vitro and in nude mouse xenografts. Oncol Rep 30: 1782-1792, 2013.

5. Shie JL, Chen ZY, Fu M, Pestell RG and Tseng CC: Gut-enriched Krüppel-like factor represses cyclin D1 promoter activity through Sp1 motif. Nucleic Acids Res 28: 2969-2976, 2000.

6. Shen L, Qu X, Ma Y, Zheng J, Chu D, Liu B, Li X, Wang M, $\mathrm{Xu}$ C, Liu N, et al: Tumor suppressor NDRG2 tips the balance of oncogenic TGF- $\beta$ via EMT inhibition in colorectal cancer. Oncogenesis 3: e86, 2014.

7. Deng Y, Yao L, Chau L, Ng SS, Peng Y, Liu X, Au WS, Wang J, $\mathrm{Li} \mathrm{F}$, Ji S, et al: N-Myc downstream-regulated gene 2 (NDRG2) inhibits glioblastoma cell proliferation. Int J Cancer 106: 342-347, 2003.

8. Xu X, Li J, Sun X, Guo Y, Chu D, Wei L, Li X, Yang G, Liu X, Yao L, et al: Tumor suppressor NDRG2 inhibits glycolysis and glutaminolysis in colorectal cancer cells by repressing c-Myc expression. Oncotarget 6: 26161-26176, 2015 .

9. Liu N, Wang L, Li X, Yang Q, Liu X, Zhang J, Zhang J, Wu Y, Ji S, Zhang Y, et al: N-Myc downstream-regulated gene 2 is involved in p53-mediated apoptosis. Nucleic Acids Res 36: 5335-5349, 2008.

10. Liu N, Wang L, Liu X, Yang Q, Zhang J, Zhang W, Wu Y, Shen L, Zhang Y, Yang A, et al: Promoter methylation, mutation, and genomic deletion are involved in the decreased NDRG2 expression levels in several cancer cell lines. Biochem Biophys Res Commun 358: 164-169, 2007.

11. Wang L, Liu N, Yao L, Li F, Zhang J, Deng Y, Liu J, Ji S, Yang A, Han $\mathrm{H}$, et al: NDRG2 is a new HIF-1 target gene necessary for hypoxia-induced apoptosis in A549 cells. Cell Physiol Biochem 21: 239-250, 2008.

12. Zhang J, Li F, Liu X, Shen L, Liu J, Su J, Zhang W, Deng Y, Wang L, Liu N, et al: The repression of human differentiationrelated gene NDRG2 expression by Myc via Miz-1-dependent interaction with the NDRG2 core promoter. J Biol Chem 281: 39159-39168, 2006.

13. Majors BS, Chiang GG and Betenbaugh MJ: Protein and genome evolution in Mammalian cells for biotechnology applications. Mol Biotechnol 42: 216-223, 2009.

14. Consortium EP; ENCODE Project Consortium: An integrated encyclopedia of DNA elements in the human genome. Nature 489: 57-74, 2012 
15. Svensson E, Vidovic K, Olofsson T, Vallon-Christersson J, Borg A and Gullberg U: The Wilms' tumor gene 1 (WT1) induces expression of the $\mathrm{N}$-myc downstream regulated gene 2 (NDRG2). DNA Cell Biol 26: 589-597, 2007.

16. Yu T, Chen X, Zhang W, Li J, Xu R, Wang TC, Ai W and Liu C: Krüppel-like factor 4 regulates intestinal epithelial cell morphology and polarity. PLoS One 7: e32492, 2012.

17. Tabuchi Y, Takasaki I, Doi T, Ishii Y, Sakai H and Kondo T: Genetic networks responsive to sodium butyrate in colonic epithelial cells. FEBS Lett 580: 3035-3041, 2006.

18. Li D, Mei H, Pu J, Xiang X, Zhao X, Qu H, Huang K, Zheng L and Tong Q: Intelectin 1 suppresses the growth, invasion and metastasis of neuroblastoma cells through up-regulation of N-myc downstream regulated gene 2. Mol Cancer 14: 47, 2015.

19. Adam PJ, Regan CP, Hautmann MB and Owens GK: Positiveand negative-acting Kruppel-like transcription factors bind a transforming growth factor beta control element required for expression of the smooth muscle cell differentiation marker SM22alpha in vivo. J Biol Chem 275: 37798-37806, 2000.

20. Bourillot PY and Savatier P: Krüppel-like transcription factors and control of pluripotency. BMC Biol 8: 125, 2010.

21. Pearson R, Fleetwood J, Eaton S, Crossley M and Bao S: Krüppel-like transcription factors: A functional family. Int J Biochem Cell Biol 40: 1996-2001, 2008.

22. Yamada T, Park CS, Mamonkin M and Lacorazza HD: Transcription factor ELF4 controls the proliferation and homing of $\mathrm{CD}^{+} \mathrm{T}$ cells via the Krüppel-like factors KLF4 and KLF2. Nat Immunol 10: 618-626, 2009.

23. Yet SF, McA'Nulty MM, Folta SC, Yen HW, Yoshizumi M Hsieh CM, Layne MD, Chin MT, Wang H, Perrella MA, et al Human EZF, a Krüppel-like zinc finger protein, is expressed in vascular endothelial cells and contains transcriptional activation and repression domains. J Biol Chem 273: 1026-1031, 1998.

24. Katz JP, Perreault N, Goldstein BG, Lee CS, Labosky PA, Yang VW and Kaestner KH: The zinc-finger transcription factor Klf4 is required for terminal differentiation of goblet cells in the colon. Development 129: 2619-2628, 2002.

25. Yu F, Li J, Chen H, Fu J, Ray S, Huang S, Zheng H and Ai W: Kruppel-like factor 4 (KLF4) is required for maintenance of breast cancer stem cells and for cell migration and invasion. Oncogene 30: 2161-2172, 2011.
26. Li T, Lu H, Shen C, Lahiri SK, Wason MS, Mukherjee D, Yu L and Zhao J: Identification of epithelial stromal interaction 1 as a novel effector downstream of Krüppel-like factor 8 in breast cancer invasion and metastasis. Oncogene 33: 4746-4755, 2014.

27. Shum CK, Lau ST, Tsoi LL, Chan LK, Yam JW, Ohira M, Nakagawara A, Tam PK and Ngan ES: Krüppel-like factor 4 (KLF4) suppresses neuroblastoma cell growth and determines non-tumorigenic lineage differentiation. Oncogene 32: 4086-4099, 2013.

28. Zhang L, Wali A, Ramana CV and Rishi AK: Cell growth inhibition by okadaic acid involves gut-enriched Kruppel-like factor mediated enhanced expression of c-Myc. Cancer Res 67: 10198-10206, 2007.

29. Ghaleb AM, McConnell BB, Nandan MO, Katz JP, Kaestner KH and Yang VW: Haploinsufficiency of Krüppel-like factor 4 promotes adenomatous polyposis coli dependent intestinal tumorigenesis. Cancer Res 67: 7147-7154, 2007.

30. Lorentzen A, Vogel LK, Lewinsky RH, Saebø M, Skjelbred CF, Godiksen S, Hoff G, Tveit KM, Lothe IM, Ikdahl T, et al: Expression of NDRG2 is down-regulated in high-risk adenomas and colorectal carcinoma. BMC Cancer 7: 192, 2007.

31. Takahashi K, Yamada M, Ohata H, Honda K and Yamada M: Ndrg2 promotes neurite outgrowth of NGF-differentiated PC12 cells. Neurosci Lett 388: 157-162, 2005.

32. Lee DC, Kang YK, Kim WH, Jang YJ, Kim DJ, Park IY, Sohn BH, Sohn HA, Lee HG, Lim JS, et al: Functional and clinical evidence for NDRG2 as a candidate suppressor of liver cancer metastasis. Cancer Res 68: 4210-4220, 2008.

33. Zhao H, Zhang J, Lu J, He X, Chen C, Li X, Gong L, Bao G, Fu Q, Chen S, et al: Reduced expression of N-Myc downstreamregulated gene 2 in human thyroid cancer. BMC Cancer 8: 303, 2008.

34. Shi H, Li N, Li S, Chen C, Wang W, Xu C, Zhang J, Jin H, Zhang $\mathrm{H}$, Zhao $\mathrm{H}$, et al: Expression of NDRG2 in esophageal squamous cell carcinoma. Cancer Sci 101: 1292-1299, 2010.

35. Chu D, Zhang Z, Li Y, Wu L, Zhang J, Wang W and Zhang J: Prediction of colorectal cancer relapse and prognosis by tissue mRNA levels of NDRG2. Mol Cancer Ther 10: 47-56, 2011. 